\title{
COHERENT GROUP RINGS AND FINITENESS CONDITIONS FOR CW-COMPLEXES
}

\author{
PHILIP S. HIRSCHHORN
}

\begin{abstract}
We characterize the class of groups $G$ that have the property that if $X$ is any space for which $\pi_{1} X \cong G$, then $X$ is homotopy equivalent to a space with finite skeleta in the "stable range" if and only if the homotopy groups of $X$ are finitely presented $Z[G]$-modules in this range. This class of groups includes all finite groups, finitely generated abelian groups, finitely generated nilpotent groups, finitely generated free groups, and free products of any of these.
\end{abstract}

We will say that a group $G$ is coherent if $G$ is finitely presented and if $Z[G]$, the integral group ring of $G$, is a coherent ring (see below). This class of groups includes, for example, all finite groups, finitely generated abelian groups, finitely generated nilpotent groups, finitely generated free groups, and free products of any of these (see [1] and [4, p. 136]). The following theorem was proved implicitly in [2].

THEOREM 1. Let $G$ be a coherent group. If $n$ is a positive integer and $X$ is a space for which $\pi_{i} X \cong \pi_{i} K(G, 1)$ for $i \leqslant n$, then

(1) If $X$ has a finite $(2 n+1)$-skeleton, then $\pi_{i} X$ is a finitely presented $Z[G]$-module for $n+1 \leqslant i \leqslant 2 n$.

(2) If $\pi_{i} X$, as a $Z[G]-$ module, is finitely presented for $n+1 \leqslant i \leqslant 2 n$ and finitely generated for $i=2 n+1$, then $X$ is homotopy equivalent to a space with a finite $(2 n+1)$-skeleton.

The purpose of this note is to state this theorem explicitly, and to prove the following converse.

THEOREM 2. If $G$ is a group for which there is an integer $n \geqslant 2$ for which the conclusions of Theorem 1 are valid, then $G$ is coherent.

This of course implies that the conclusions of Theorem 1 are then valid for any positive integer $n$. I have not been able to characterize the class of groups for which Theorem 1 holds with $n=1$; this class certainly includes the coherent groups, but might possibly include some noncoherent groups as well.

Definition. A ring $R$ is coherent if every homomorphism between finitely generated free modules has a finitely generated kernel.

Received by the editors June 2, 1978.

AMS (MOS) subject classifications (1970). Primary 55D99.

(C) 1979 American Mathematical Society 0002-9939/79/0000-0233/\$01.75 
The class of coherent rings thus includes the Noetherian rings. Coherent group rings also have important applications in algebraic $K$-theory (see [5]).

Before giving the proof of Theorem 2, we give the following slight refinement of Theorem 1 .

THEOREM 1'. Let $G$ be a coherent group. If $n$ is a positive integer and $X$ is a space for which $\pi_{i} X \cong \pi_{i} K(G, 1)$ for $i \leqslant n$, then for any integer $k$ in the range $1 \leqslant k<2 n+1$ the following two conditions are equivalent:

(1) $X$ is homotopy equivalent to a space with a finite $k$-skeleton.

(2) As a $Z[G]$-module, $\pi_{i} X$ is finitely presented for $n+1 \leqslant i<k$ and finitely generated for $i=k$.

If $k=2 n+1$, then (2) implies (1), and (1) implies that $\pi_{i} X$ is finitely presented for $n+1 \leqslant i \leqslant 2 n$.

The proof of Theorem $1^{\prime}$ is similar to the proof of Theorem 1 . When $k<2 n+1$, one shows that (1) implies (2) by showing that $\pi_{i} X^{k}$ is finitely presented for $i \leqslant k$ (where $X^{k}$ is the $k$-skeleton of $X$ ). Since $\pi_{k} X$ is a quotient of $\pi_{k} X^{k}$, this implies that $\pi_{k} X$ is a finitely generated $Z[G]$-module. Theorem $1^{\prime}$ is the best possible result, as can be seen by considering the wedge $W=S^{1} \vee S^{n+1}$, which is a finite complex with $\pi_{2 n+1} W$ not finitely generated over $Z\left[\pi_{1} W\right]$.

Proof of Theorem 2. Since $G$ satisfies part (2) of Theorem 1, we can take a $K(G, 1)$ with a finite $(2 n+1)$-skeleton. This implies that $G$ is finitely presented, and so it remains only to show that $Z[G]$ is a coherent ring. Let $f$ : $F \rightarrow \bar{F}$ be a homomorphism of $Z[G]$-modules with $F$ and $\bar{F}$ free of finite ranks $s$ and $t$, respectively. We must show that the kernel of $f$ is finitely generated.

Let $W$ be the wedge of our $K(G, 1)$ with $t$ copies of the $(n+1)$-sphere. Then [6, Theorem 19] $\pi_{n+1} W=\bar{F}$ as $Z[G]$-modules, and we can attach $s$ different $(n+2)$-cells to $W$, with attaching maps determined by the homomorphism $f$, and obtain a complex $X$. Now $X$ is a complex with a finite $(2 n+1)$-skeleton, and $\pi_{i} X \cong \pi_{i} K(G, 1)$ for $i \leqslant n$. Since $G$ satisfies part (1) of Theorem 1 , this implies that $\pi_{n+2} X$ is a finitely presented $Z[G]$-module.

Now consider $\tilde{X}$, the universal cover of $X$. The chain complex of $\tilde{X}$ consists of free $Z[G]$-modules with one generator for each cell of $X$, and it is not hard to see that $H_{n+2} \tilde{X}$ is isomorphic to the kernel of $f$. Since $\tilde{X}$ is $n$-connected, the Hurewicz theorem [3, p. 167] now gives an isomorphism $\pi_{n+1} X \cong H_{n+1} \tilde{X}$ and an epimorphism $\pi_{n+2} X \rightarrow H_{n+2} \tilde{X}$, and so $H_{n+2} \tilde{X}$ is a finitely generated $Z[G]$-module, and the proof of Theorem 2 is complete.

\section{BIBLIOGRAPHY}

1. K. G. Choo, K. Y. Lam and E. Luft, On free products of rings and the coherence property, Algebraic $K$-Theory. II, H. Bass (editor), Lecture Notes in Math., vol. 342, Springer-Verlag, Berlin, 1973.

2. P. S. Hirschhorn, Link complements and coherent group rings, Illinois J. Math. (to appear). 
3. S. T. Hu, Homotopy theory, Academic Press, New York, 1959.

4. D. S. Passman, Infinite group rings, Marcel Dekker, New York, 1971.

5. F. Waldhausen, Whitehead groups of generalized free products, Algebraic K-Theory. II, H. Bass (editor), Lecture Notes in Math., vol. 342, Springer-Verlag, Berlin, 1973.

6. J. H. C. Whitehead, Simplicial spaces, nuclei, and m-groups, Proc. London Math. Soc. (2) 45 (1939), 243-327.

Department of Mathematics, Brandeis University, Waltham, Massachusetts 02154

Current address: Department of Mathematics, Amherst College, Amherst, Massachusetts 01002 